DFTT 20/2003

hep-th/0310157

\title{
Non-Perturbative Gauge Superpotentials from Supergravity
}

\author{
Emiliano Imeroni ${ }^{a, c}$ and Alberto Lerda ${ }^{b, a, c}$ \\ a Dipartimento di Fisica Teorica, Università di Torino, \\ Via P. Giuria 1, I-10125 Torino, Italy \\ ${ }^{b}$ Dipartimento di Scienze e Tecnologie Avanzate \\ Università del Piemonte Orientale, I-15100 Alessandria, Italy \\ ${ }^{c}$ I.N.F.N., Sezione di Torino, Via P. Giuria 1, I-10125 Torino, Italy \\ email: imeroni@to.infn.it, lerda@to.infn.it
}

\begin{abstract}
We study $U(N)$ SQCD with $N_{f} \leq N$ flavors of quarks and antiquarks by engineering it with a configuration of fractional D3-branes on a $\mathbb{C}^{3} / \mathbb{Z}_{2} \times \mathbb{Z}_{2}$ orbifold. In particular we show how the moduli space of the gauge theory naturally emerges from the classical geometry produced by the D3-branes, and how the non-perturbatively generated superpotential is recovered from geometrical data.
\end{abstract}




\section{Contents}

1 Introduction 1

2 SQCD from fractional branes on $\mathbb{C}^{3} / \mathbb{Z}_{2} \times \mathbb{Z}_{2} \quad 2$

3 Summary of the supergravity solution 44

4 The dual gauge theory and its classical moduli space 6

5 The ADS superpotential 10

$\begin{array}{ll}\text { References } & 14\end{array}$

\section{Introduction}

The search for extensions of the gauge/gravity correspondence towards non-conformal theories with a reduced number of supercharges has recently achieved some interesting progress. Indeed, it has been shown that many relevant properties of non-conformal $\mathcal{N}=1$ and $\mathcal{N}=2$ supersymmetric gauge theories in four dimensions can be extracted from dual supergravity solutions associated to different set-ups of strings and D-branes. For example, at the perturbative level one can get the correct logarithmic running of the coupling constant and the chiral anomaly, while at the non-perturbative level one can discuss instantons, gaugino condensation and confining strings.

Many of these results can be obtained by studying in detail the supergravity solutions produced by stacks of fractional D3-branes in conifold [1, 2, 3, 4, 15] and orbifold [1, 6, 7. 8] backgrounds. Alternatively, non-conformal gauge theories with $\mathcal{N}=1$ or $\mathcal{N}=2$ supersymmetry can be realized by wrapping D-branes on suitable supersymmetric cycles in Calabi-Yau or K3 manifolds [9]. Also in these cases, a detailed study of the corresponding supergravity solutions has provided relevant information on the dual gauge theory [4, 10]. Most of these developments are covered in recent reviews [11, 12] to which we refer also for a more extended bibliography.

Another line of research has been the study of the so-called "geometric transitions" [13, 14], where one engineers gauge theories by wrapping D5-branes on two-cycles of resolved Calabi-Yau manifolds in such a way that the geometry flows to a deformed manifold where branes are replaced by fluxes through the newly blown-up three-cycles. In this framework, it has been shown [15, 16] how to get the non-perturbatively generated effective superpo- 
tential of the dual $\mathcal{N}=1$ gauge theory [17, 14] by means of geometric considerations.

In this paper we bring together these lines of research, and show that the explicit knowledge of the supergravity solution produced by a stack of D-branes can be fruitfully combined with general geometric considerations on the background in which they are embedded. In this way one can obtain relevant information on the dual gauge theory, both at a perturbative and at a non-perturbative level. In particular we will discuss the AffleckDine-Seiberg theory, namely $U(N) \mathcal{N}=1$ Super QCD with $N_{f}<N$ flavors of quarks and antiquarks, which we realize as the world-volume theory of a stack of fractional D3-branes on a $\mathbb{C}^{3} / \mathbb{Z}_{2} \times \mathbb{Z}_{2}$ orbifold. We then show how to use this fractional brane configuration to obtain the running coupling constant, the classical moduli space of the low-energy theory and the non-perturbative effective superpotential [18] of SQCD in the chosen regime. We also comment on SQCD with $N=N_{f}$ which simply arises as a particular case of our construction.

This paper is organized as follows. In section 2 we introduce the $\mathbb{C}^{3} / \mathbb{Z}_{2} \times \mathbb{Z}_{2}$ orbifold and show how $U(N)$ SQCD can be engineered via a specific configuration of fractional D3-branes. After summarizing in section 3 the corresponding supergravity solution, in section 4 we study the dual gauge theory, deriving the running gauge coupling constant and exploring the classical moduli space. Finally, in section 5 we show how the nonperturbatively generated Affleck-Dine-Seiberg superpotential can be obtained by the fluxes of the dual supergravity solution together with some geometric considerations.

\section{SQCD from fractional branes on $\mathbb{C}^{3} / \mathbb{Z}_{2} \times \mathbb{Z}_{2}$}

The system we are going to consider is a stack of fractional D3-branes on the orbifold $\mathbb{R}^{1,3} \times \mathbb{C}^{3} / \mathbb{Z}_{2} \times \mathbb{Z}_{2}[19$. In this space, which is a singular and non-compact Calabi-Yau three-fold, a stack of D-branes will preserve four supercharges. In particular we arrange the fractional branes according to Table 1 where - and · indicate, respectively, longitudinal and transverse directions.

\begin{tabular}{|c|c|c|c|c|c|c|c|c|c|c|}
\hline & 0 & 1 & 2 & 3 & 4 & 5 & 6 & 7 & 8 & 9 \\
\hline D3 & - & - & - & - & $\cdot$ & $\cdot$ & $\cdot$ & $\cdot$ & $\cdot$ & $\cdot$ \\
\hline
\end{tabular}

Table 1: Fractional D3-branes on $\mathbb{C}^{3} / \mathbb{Z}_{2} \times \mathbb{Z}_{2}$.

In the following we will denote with $x^{\alpha}(\alpha=0, \ldots, 3)$ the coordinates transverse to 


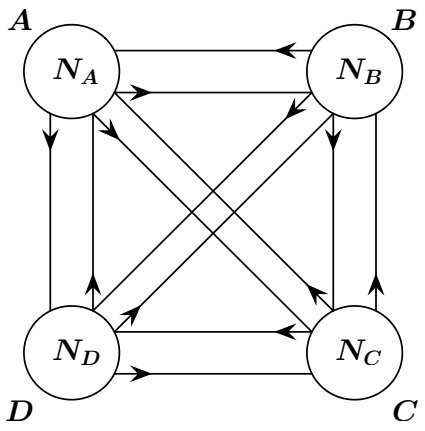

Figure 1: Each node $i$ of the quiver diagram represents a gauge group factor $U\left(N_{i}\right)$ with the corresponding vector multiplet, and each oriented arrow from node $i$ to node $j$ represents a chiral multiplet in the $\left(\mathbf{N}_{\mathbf{i}}, \overline{\mathbf{N}}_{\mathbf{j}}\right)$ representation.

the orbifold, and introduce three complex coordinates in the orbifolded directions $x^{r}(r=$ $4, \ldots, 9)$ as follows

$$
z_{1}=x^{4}+i x^{5}, \quad z_{2}=x^{6}+i x^{7}, \quad z_{3}=x^{8}+i x^{9} .
$$

The generators of the two $\mathbb{Z}_{2}$ factors of the orbifold group are denoted by $g_{1}$ and $g_{2}$, and their action on the complex coordinates is given by

\begin{tabular}{c|ccc} 
& $z_{1}$ & $z_{2}$ & $z_{3}$ \\
\hline$g_{1}$ & $z_{1}$ & $-z_{2}$ & $-z_{3}$ \\
$g_{2}$ & $-z_{1}$ & $z_{2}$ & $-z_{3}$
\end{tabular}

The remaining two elements of the orbifold group are of course the identity $e$ and $g_{3}=g_{1} g_{2}$.

As is well known, the most elementary configurations of branes on orbifolds are made by fractional branes [20]. These are defined by the fact that the Chan-Paton factors of the open strings attached to them transform in the irreducible representations of the orbifold group; moreover, they have the property of being stuck at the orbifold fixed point. In our $\mathbb{Z}_{2} \times \mathbb{Z}_{2}$ orbifold, we then have four different types of fractional D3-branes, that we denote as A, B, C, D, corresponding to the four irreducible one-dimensional representations of the orbifold group. The low-energy theory living on a generic system of $N_{k}$ D3-branes of type $k$, is a four-dimensional $\mathcal{N}=1$ gauge theory with gauge group $U\left(N_{\mathrm{A}}\right) \times U\left(N_{\mathrm{B}}\right) \times$ $U\left(N_{\mathrm{C}}\right) \times U\left(N_{\mathrm{D}}\right)$ and twelve chiral multiplets, which transform in the fundamental (or antifundamental) representation of a particular gauge group factor and carry a flavor index with respect to the other three factors. All this information can be encoded in the quiver diagram represented in Fig. 1].

Our goal is to use this orbifold set-up to engineer $U(N) \mathcal{N}=1$ SQCD, which is a theory with $N_{f}$ flavors of "quarks" and "antiquarks". A possibility to do this would be to consider 


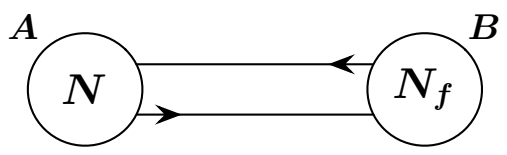

Figure 2: The quiver diagram associated to a system of $N$ fractional D3-branes of type A and $N_{f}$ fractional D3-branes of type B which is used to engineer $U(N)$ SQCD with $N_{f}$ flavors.

a stack of $N$ fractional D3-branes of a given type, and add to them $N_{f}$ D7-branes in order to introduce the fundamental and anti-fundamental chiral multiplets [8]. Here, however, we will follow an alternative and simpler route [21], which allows to obtain the main features of $\mathcal{N}=1$ SQCD in a very natural way by using only D3-branes. This alternative strategy amounts simply to consider a configuration of $N$ fractional D3-branes of, say, type A and $N_{f}$ fractional D3-branes of, say, type B, which gives rise to the $U(N) \times U\left(N_{f}\right)$ gauge theory represented by the diagram of Fig. 2. From this quiver model we clearly can obtain $U(N)$ SQCD with $N_{f}$ flavors if we concentrate only on the low-energy degrees of freedom associated to the branes of type A by a suitable selection of open strings with appropriate Chan-Paton factors. Even if in the complete theory the flavor symmetry $U\left(N_{f}\right)$ is also gauged, we will see that this configuration of fractional D3 branes is able to provide a big deal of information on SQCD via the gauge/gravity correspondence.

\section{Summary of the supergravity solution}

The classical supergravity solution describing a system of $N_{k}$ fractional D3-branes of type $k$ placed at the origin $z_{1}=z_{2}=z_{3}=0$ of $\mathbb{C}^{3} / \mathbb{Z}_{2} \times \mathbb{Z}_{2}$ was constructed in Ref. [7]. Here we briefly summarize its main properties.

Let us first recall that the bosonic fields of type IIB supergravity are the metric, the 2-form $B_{2}$ and the dilaton $\phi$ from the NS-NS sector, and the 0 -form $C_{0}$, the 2 -form $C_{2}$ and the 4-form $C_{4}$ with self-dual field strength $\tilde{F}_{5}$ from the R-R sector. Then, the fractional D3brane solution is obtained by assuming that the two 2-forms $B_{2}$ and $C_{2}$ have components only along the three exceptional shrinking 2-cycles $\mathcal{C}_{i}(i=1,2,3)$ of the orbifold geometry. Specifically, one writes

$$
B_{2}=b_{i} \omega_{2}^{(i)}, \quad C_{2}=c_{i} \omega_{2}^{(i)},
$$

where the anti-self dual $(1,1)$-forms $\omega_{2}^{(i)}$, dual to the 2 -cycles $\mathcal{C}_{i}$, are defined and normalized as

$$
\int_{\mathcal{C}_{i}} \omega_{2}^{(j)}=\delta_{i}^{j}, \quad \int \omega_{2}^{(i)} \wedge \omega_{2}^{(i)}=-\frac{1}{4}, \quad{ }^{\star} \omega_{2}^{(i)}=-\omega_{2}^{(i)} .
$$


The scalar fields $b_{i}$ and $c_{i}$ in (3.1) are called twisted fields, and precisely correspond to massless degrees of freedom of the three NS-NS and R-R twisted sectors of the conformal field theory describing closed strings in the orbifold $\mathbb{C}^{3} / \mathbb{Z}_{2} \times \mathbb{Z}_{2}$.

If we define

$$
G_{3}=d C_{2}+\left(C_{0}+\mathrm{i}^{-\phi}\right) d B_{2},
$$

and

$$
\gamma_{i}=c_{i}+\mathrm{i} b_{i},
$$

then the solution for a stack of $N_{k}$ fractional D3 branes of type $k$ reads [7]

$$
\begin{aligned}
d s^{2} & =H_{3}^{-1 / 2} \eta_{\alpha \beta} d x^{\alpha} d x^{\beta}+H_{3}^{1 / 2} \delta_{r s} d x^{r} d x^{s}, \\
C_{0} & =0, \quad \mathrm{e}^{\phi}=1, \\
\tilde{F}_{5} & =d H_{3}^{-1} d x^{0} \wedge \ldots \wedge d x^{3}+{ }^{\star}\left(d H_{3}^{-1} d x^{0} \wedge \ldots \wedge d x^{3}\right), \\
G_{3} & =d \gamma_{i} \wedge \omega_{2}^{(i)} .
\end{aligned}
$$

Here the functions $\gamma_{i}$ are given by

$$
\gamma_{i}=\mathrm{i} K f_{i}\left(N_{k}\right) \ln \frac{z_{i}}{\epsilon_{0}},
$$

where $\epsilon_{0}$ is a short-distance regulator, $K=4 \pi g_{s} l_{s}^{2}$ ( $g_{s}$ being the string coupling constant and $l_{s}$ the string length) and the $f_{i}$ 's are functions of the numbers of the different types of fractional branes

$$
\begin{aligned}
& f_{1}\left(N_{k}\right)=N_{\mathrm{A}}+N_{\mathrm{B}}-N_{\mathrm{C}}-N_{\mathrm{D}}, \\
& f_{2}\left(N_{k}\right)=N_{\mathrm{A}}-N_{\mathrm{B}}+N_{\mathrm{C}}-N_{\mathrm{D}}, \\
& f_{3}\left(N_{k}\right)=N_{\mathrm{A}}-N_{\mathrm{B}}-N_{\mathrm{C}}+N_{\mathrm{D}} .
\end{aligned}
$$

Finally, $H_{3}$ is a specific function of $z_{i}$ whose explicit expression, which we will not need in the following, can be found in Ref. [7].

As is clear from the solution (3.5), each individual fractional D3-brane is charged under all three twisted sectors of the closed string theory on the orbifold $\mathbb{C}^{3} / \mathbb{Z}_{2} \times \mathbb{Z}_{2}$, as well as under the untwisted one. This is perfectly consistent with the description of fractional D-branes in terms of boundary states which represent the sources for all closed string states emitted by the D-branes. In our specific case, the boundary states for the various fractional D3-branes are schematically given by

$$
\begin{aligned}
& |\mathrm{A}\rangle=|\mathrm{U}\rangle+|\mathrm{T} 1\rangle+|\mathrm{T} 2\rangle+|\mathrm{T} 3\rangle, \\
& |\mathrm{B}\rangle=|\mathrm{U}\rangle+|\mathrm{T} 1\rangle-|\mathrm{T} 2\rangle-|\mathrm{T} 3\rangle, \\
& |\mathrm{C}\rangle=|\mathrm{U}\rangle-|\mathrm{T} 1\rangle+|\mathrm{T} 2\rangle-|\mathrm{T} 3\rangle, \\
& |\mathrm{D}\rangle=|\mathrm{U}\rangle-|\mathrm{T} 1\rangle-|\mathrm{T} 2\rangle+|\mathrm{T} 3\rangle,
\end{aligned}
$$


where $|U\rangle$ is the contribution of the untwisted sector, $|\mathrm{T} i\rangle$ is the one of the $i$-th twisted sector corresponding to the group element $g_{i}$, and the signs are consistent with (3.7).

Finally, we remark that the supergravity solution (3.5) has a naked singularity of repulson type, a common feature of all classical solutions describing fractional branes on orbifolds. One should then proceed to examine the appearance of an enhançon mechanism 22] that would make this geometry acceptable, but we will not perform this analysis here. Instead, in the following sections we will use the above solution to study the dual $\mathcal{N}=1$ gauge theory.

\section{The dual gauge theory and its classical moduli space}

Let us now concentrate on the configuration made up of $N$ fractional D3-branes of type A and $N_{f}$ fractional D3-branes of type B that we introduced in section 2 and represented in Fig. 2. As we have seen, the theory on the world-volume of the type A branes is $U(N)$ SQCD with $\mathcal{N}=1$ supersymmetry, $N_{f}$ "quark" chiral multiplets, $Q^{i}$, and $N_{f}$ "antiquark" chiral multiplets, $\tilde{Q}_{\tilde{\jmath}}$.

Many properties of this gauge theory can be explicitly obtained from the supergravity solution (3.5) (with $N_{C}=N_{D}=0$ ). As a first example, let us consider the running gauge coupling constant $g_{\mathrm{YM}}$, which, as shown in Ref. [7], can be directly related to the twisted scalars $b_{i}$ of the dual geometry, according to

$$
\frac{1}{g_{\mathrm{YM}}^{2}}=\frac{1}{8 \pi g_{s}} \frac{1}{\left(2 \pi l_{s}\right)^{2}} \sum_{i=1}^{3} b_{i}
$$

The right hand side of this equation can be equivalently written also in terms of the flux of $G_{3}$ across an appropriate singular 3-cycle of the orbifold $\mathbb{C}^{3} / \mathbb{Z}_{2} \times \mathbb{Z}_{2}$. To see this, and also for our later analysis of the superpotential, it is useful to identify the singular 3-cycles that exist in this non-compact Calabi-Yau space.

Since there are no exceptional $(1,2)$ or $(2,1)$-forms coming from the twisted sectors, everything should arise from the $(1,1)$-forms $\omega_{2}^{(i)}$, dual to the singular 2 -cycles $\mathcal{C}_{i}$ that we already encountered in section 3. Thus, we can introduce three compact 3-cycles $A_{i}$ and three non-compact 3 -cycles $B_{i}$ by simply taking the direct product of $\mathcal{C}_{i}$ with suitable 1-cycles in the $z_{i}$ planes. Specifically, we define

$$
A_{i}=\alpha_{i} \times \mathcal{C}_{i}, \quad B_{i}=\beta_{i} \times \mathcal{C}_{i} \quad(i=1,2,3),
$$

where the compact cycles $\alpha_{i}$ and the non-compact cycles $\beta_{i}$ in the $z_{i}$ plane are orthogonal to each other and are shown in Fig. 3. Using the last equation of (3.5) and the normal- 


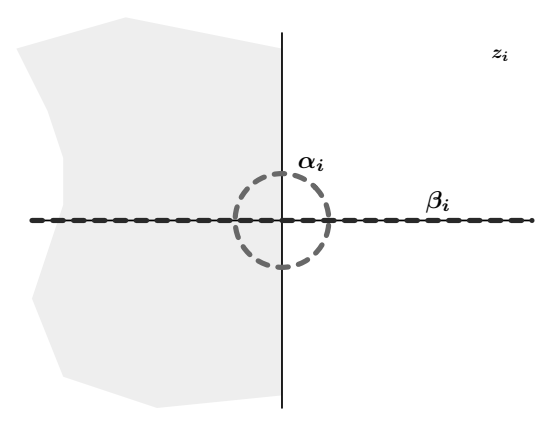

Figure 3: The compact 1-cycle $\alpha_{i}$ and the noncompact 1-cycle $\beta_{i}$ in the $z_{i}$ plane.

izations (3.2), one can easily see that the fluxes of $G_{3}$ along these 3-cycles are given by

$$
\int_{A_{i}\left(\text { or } B_{i}\right)} G_{3}=\int_{A_{i}\left(\text { or } B_{i}\right)} d \gamma_{j} \wedge \omega_{2}^{(j)}=\int_{\alpha_{i}\left(\text { or } \beta_{i}\right)} d \gamma_{i} \int_{\mathcal{C}_{i}} \omega_{2}^{(i)}=\int_{\alpha_{i}\left(\text { or } \beta_{i}\right)} d \gamma_{i} .
$$

In particular, for our specific brane configuration, we find

$$
\int_{A_{1}} G_{3}=-2 \pi K\left(N+N_{f}\right), \quad \int_{A_{2}} G_{3}=\int_{A_{3}} G_{3}=-2 \pi K\left(N-N_{f}\right),
$$

and

$$
\int_{B_{1}} G_{3}=\mathrm{i} K\left(N+N_{f}\right) \ln \frac{\rho_{c}}{\rho_{0}}, \quad \int_{B_{2}} G_{3}=\int_{B_{3}} G_{3}=\mathrm{i} K\left(N-N_{f}\right) \ln \frac{\rho_{c}}{\rho_{0}} .
$$

In the last line, the integrations over $\rho_{i}=\left|z_{i}\right|$ in the non-compact cycles $\beta_{i}$ extend up to a cut-off $\rho_{c}$, which sets the higher scale, while the lower scale $\rho_{0}$ is introduced as a further short-distance cut-off, since the validity of the singular supergravity solution stops at a finite distance from the brane position.

If we identify $\rho_{0}$ with the (arbitrary) cut-off $\epsilon_{0}$ appearing in (3.6), we immediately see that $b_{i}=-\mathrm{i} \int_{B_{i}} G_{3}$, and thus (4.1) becomes

$$
\frac{1}{g_{\mathrm{YM}}^{2}}=\frac{1}{8 \pi g_{s}} \frac{1}{\left(2 \pi l_{s}\right)^{2}} \sum_{i=1}^{3}\left(-\mathrm{i} \int_{B_{i}} G_{3}\right)=\frac{1}{8 \pi^{2}}\left(3 N-N_{f}\right) \ln \frac{\rho_{c}}{\rho_{0}} .
$$

Introducing the renormalization scale $\mu$ and the dynamically generated scale $\Lambda$ through the usual energy/radius relations

$$
\rho_{c}=2 \pi l_{s}^{2} \mu, \quad \rho_{0}=2 \pi l_{s}^{2} \Lambda,
$$

that follow from a "stretched string" analysis [23], we easily get from (4.6) the correct one-loop running coupling constant for our $U(N)$ SQCD theory. 
We now turn to the vacuum structure of this theory and show how it can be recovered from the supergravity solution (3.5) of fractional D3-branes in a very simple way. Since it is known that $U(N)$ SQCD has a very different behavior depending on the number of flavors (see for instance Ref. 24]), here we concentrate on the case in which the number of flavors $N_{f}$ is less than the number of colors $N$. This is the so-called Affleck-Dine-Seiberg (ADS) theory [18, in which an effective superpotential is generated by non-perturbative effects.

To see how the vacuum structure of the ADS theory can be recovered from supergravity, let us begin by considering a single fractional D3-brane of type A together with one of type B. As individual objects, these branes are charged under all four sectors of closed string theory, and for this reason they cannot move off the orbifold fixed point (see the corresponding boundary states (3.8) ). However, since A and B are mutually BPS objects, we can easily construct the superposition $\mathrm{A}+\mathrm{B}$, which will be charged under the sector twisted by $g_{1}$ (with a charge double with respect to a single fractional brane), but will not carry any charge under the other two twisted sectors. This can be easily seen in the boundary state description, where one writes

$$
|\mathrm{A}+\mathrm{B}\rangle=|\mathrm{A}\rangle+|\mathrm{B}\rangle=2(|\mathrm{U}\rangle+|\mathrm{T} 1\rangle)
$$

Thus, the superposition $\mathrm{A}+\mathrm{B}$ can freely move in the $z_{1}$ plane, which is left fixed by the action of $g_{1}$. Notice that when the pair $\mathrm{A}+\mathrm{B}$ is not at the origin, it cannot be divided anymore into its components, which indeed are defined only at the orbifold fixed point. The crucial observation is that the motion of $\mathrm{A}+\mathrm{B}$ causes the breaking of the $U(1) \times U(1)$ gauge group of the theory living on the superposition down to $U(1)$ via the Higgs mechanism.

Returning to our SQCD configuration and repeating the above argument, one finds that out of the $N$ branes of type A and the $N_{f}$ branes of type B, it is possible to build $N_{f} \mathrm{~A}+\mathrm{B}$ superpositions and move them away from the origin at arbitrary points in the $z_{1}$ plane. The motion of these $\mathrm{A}+\mathrm{B}$ pairs is naturally interpreted as giving arbitrary vacuum expectation values to the meson matrix $M_{\tilde{\jmath}}^{i}=Q^{i} \tilde{Q}_{\tilde{\jmath}}$, thus breaking the gauge group $U(N)$ down to $U\left(N-N_{f}\right)$ corresponding to the world-volume theory of remaining $N-N_{f}$ fractional branes of type A still placed at the origin. Therefore, this D-brane construction uncovers the correct classical moduli space of the ADS theory in a very natural way, as shown in Fig. 4a. This same mechanism works also for $N=N_{f}$. In this case, all branes can form pairs and, since no unpaired A branes are left, no unbroken gauge theory remains. Notice that this description makes very clear that something drastic happens when passing from $N_{f} \leq N$ to $N_{f}>N$, in agreement with the known field theory results [24]. Indeed, if $N_{f}>N$, all we have done is no longer valid and one is forced to look for some alternative description.

Let us now discuss how the supergravity solution (3.5) gets modified when we form the 
a)

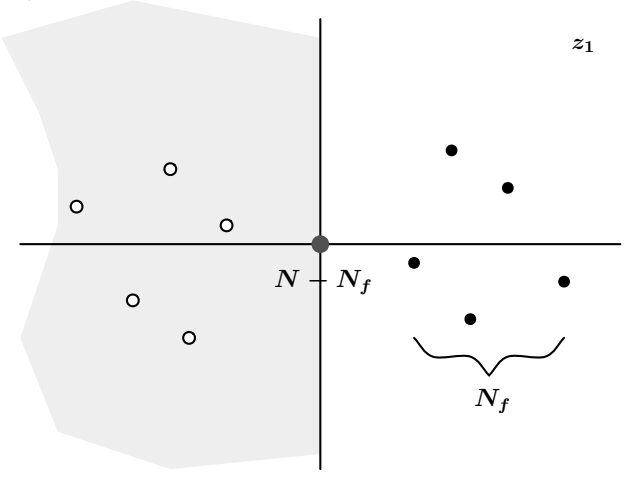

b)

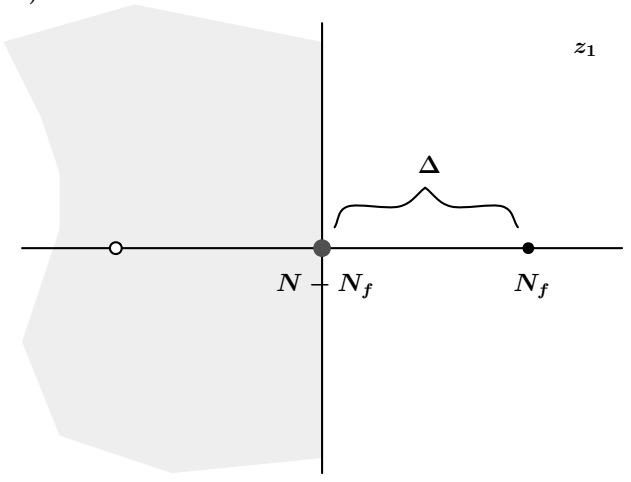

Figure 4: Moduli space of the ADS theory via fractional branes. a) A+B superpositions at arbitrary points of the $z_{1}$ plane, together with their images on the covering space. $b$ ) The configuration which makes the meson matrix $M_{\tilde{\jmath}}^{i}$ proportional to the identity.

$\mathrm{A}+\mathrm{B}$ superpositions and move them in the $z_{1}$ plane. For simplicity, but without any loss of generality, we place all $N_{f}$ superpositions at the same point $z_{1}=\Delta$ on the real axis of the $z_{1}$ plane, as shown in Fig. 4b (clearly, in order to have an even configuration under the orbifold group, we also need to put images at the identified point $z_{1}=-\Delta$ ). This set-up makes the meson matrix proportional to the identity, $M_{\tilde{\jmath}}^{i}=m^{2} \delta_{\tilde{\jmath}}^{i}$, where $m$ is related to $\Delta$ via the usual gauge/gravity identification

$$
\Delta=2 \pi l_{s}^{2} m
$$

We will be interested in the case in which $\Delta$ and $m$ are very large.

As we have seen before, what is relevant for the dual gauge theory is the knowledge of the twisted fields $\gamma_{i}$, which now become

$$
\gamma_{i}=\mathrm{i} K\left[\left(N-N_{f}\right) \ln \frac{z_{i}}{\epsilon_{0}}+\delta_{i, 1} N_{f} \ln \frac{z_{1}-\Delta}{\epsilon_{0}}+\delta_{i, 1} N_{f} \ln \frac{z_{1}+\Delta}{\epsilon_{0}}\right] \text {. }
$$

Then, using (4.2) and (4.3), we find that the flux of $G_{3}$ along the $A_{i}$ cycles is given by

$$
\int_{A_{i}} G_{3}=-2 \pi K\left(N-N_{f}\right)
$$

for all $i$ 's, while the flux along the $B_{i}$ cycles becomes

$$
\int_{B_{i}} G_{3}=\mathrm{i} K\left[\left(N-N_{f}\right) \ln \frac{\rho_{c}}{\rho_{0}}+\delta_{i, 1} 2 N_{f} \ln \frac{\Delta}{\rho_{0}}\right],
$$


where we have assumed $\rho_{c} \ll \Delta$ and denoted again by $\rho_{0}$ the minimum distance (from the branes at the origin and the pairs in $\pm \Delta$ ) that one can reach along the integration path on $\rho_{i}=\left|z_{i}\right|$ in the $\beta_{i}$ cycles. $^{1}$

Thus, the gauge coupling constant for the world-volume theory of the unpaired A branes is

$$
\frac{1}{g_{\mathrm{YM}}^{2}}=\frac{1}{8 \pi g_{s}} \frac{1}{\left(2 \pi l_{s}\right)^{2}} \sum_{i=1}^{3}\left(-\mathrm{i} \int_{B_{i}} G_{3}\right)=\frac{3}{8 \pi^{2}}\left(N-N_{f}\right) \ln \frac{\rho_{c}}{\rho_{0}}+\frac{2 N_{f}}{8 \pi^{2}} \ln \frac{\Delta}{\rho_{0}}
$$

which in terms of the gauge theory scales becomes

$$
\frac{1}{g_{\mathrm{YM}}^{2}}=\frac{3}{8 \pi^{2}}\left(N-N_{f}\right) \ln \frac{\mu}{\Lambda}+\frac{2 N_{f}}{8 \pi^{2}} \ln \frac{m}{\Lambda},
$$

with $\mu \ll m$. The above coupling can be expressed also in terms of the low-energy effective unbroken $U\left(N-N_{f}\right)$ theory as

$$
\frac{1}{g_{\mathrm{YM}}^{2}}=\frac{3}{8 \pi^{2}}\left(N-N_{f}\right) \ln \frac{\mu}{\Lambda_{L}}
$$

where the low-energy scale $\Lambda_{L}$ is related to $\Lambda$ and $m$ via

$$
\Lambda_{L}^{3\left(N-N_{f}\right)}=\frac{\Lambda^{3 N-N_{f}}}{m^{2 N_{f}}}=\frac{\Lambda^{3 N-N_{f}}}{\operatorname{det} M}
$$

We then see that the supergravity computation precisely reproduces, beyond the running coupling constant, also the expected matching of scales in the gauge theory [24]. This concludes our analysis of the theory at the classical and perturbative levels. In the next section, we will turn to non-perturbative phenomena, with the computation of the effective superpotential.

\section{The ADS superpotential}

We now study the quantum moduli space of SQCD using our D-brane set-up, and see that our quantitative results perfectly agree with the field theory analysis, predicting that the classical moduli space is lifted by the generation of the ADS superpotential [18.

The main ingredient we will implement is the formula [17, 14] that, in the cases where the dilaton is constant, expresses the $\mathcal{N}=1$ effective gauge superpotential $W_{\text {eff }}$ in terms

\footnotetext{
${ }^{1}$ Notice that if instead $\rho_{c} \gg \Delta$, the flux of $G_{3}$ along the $B_{i}$ cycles is given by (4.5), in agreement with the fact that in this case all degrees of freedom are light and thus contribute to the running of the coupling constant as in (4.6).
} 
of the fluxes of the complex three-form $G_{3}$ of the dual IIB supergravity solution and the periods of the holomorphic $(3,0)$-form $\Omega$ of the Calabi-Yau orbifold in which the branes are put, namely

$$
W_{\mathrm{eff}} \propto \sum_{i}\left[\int_{A_{i}} G_{3} \int_{B_{i}} \Omega-\int_{A_{i}} \Omega \int_{B_{i}} G_{3}\right] .
$$

As a brief summary, we recall that this formula has been shown to give the correct answer in many set-ups where the gauge theory is engineered via D-brane configurations on CalabiYau manifolds which undergo a geometric transition, where some 3-cycles of the manifold blow up and the branes are replaced by fluxes of IIB supergravity fields [13, 14]. For example, using this approach the Veneziano-Yankielowicz superpotential [25] of pure $\mathcal{N}=$ 1 super Yang-Mills theory has been extracted [15, 26] from the warped deformed conifold solution by Klebanov and Strassler [3].

In the case at hand, the supergravity solution we have at our disposal is not smooth, unlike the solution of Ref. [3]. Rather, we are in a situation, the orbifold limit, where all 2- and 3-cycles are shrinking, similarly to what happens in the singular conifold solution of Klebanov and Tseytlin [2]. However, this does not seem to be an obstacle for using (5.1). In fact, in the conifold case the knowledge of the singular Klebanov-Tseytlin solution is sufficient for computing the $G_{3}$ fluxes that are needed in (5.1), for the very simple reason that they are precisely identified with the fluxes of the regular Klebanov-Strassler solution. ${ }^{2}$ The same is true also in our case, and thus even if we do not know a regular solution in a deformed orbifold, the $G_{3}$ fluxes can be obtained from the singular solution as described in section 4. On the other hand, the periods of $\Omega$ that enter in (5.1) cannot be determined in the singular case, since they crucially depend on the details of the deformation of the geometry. However, the geometric considerations which are necessary for getting the correct periods do not depend on the details of the classical solution and can be worked out in full generality also for the orbifold $\mathbb{C}^{3} / \mathbb{Z}_{2} \times \mathbb{Z}_{2}$. Thus, we can fruitfully combine the knowledge of the fluxes coming from our explicit supergravity solution with the geometric features of the deformed background. This is the approach we take with our fractional D-branes in the orbifold $\mathbb{C}^{3} / \mathbb{Z}_{2} \times \mathbb{Z}_{2}$.

We already derived the fluxes of $G_{3}$ along the $A_{i}$ and $B_{i}$ cycles: they are given in (4.11) and (4.12). Let us then consider the periods of the holomorphic $(3,0)$-form $\Omega$. As in the case of the conifold, in order to get sensible results it is necessary to deform the singular geometry of the orbifold. Let us start by noting that the space $\mathbb{C}^{3} / \mathbb{Z}_{2} \times \mathbb{Z}_{2}$ can be described as the $F(x, y, z, t)=0$ hypersurface in $\mathbb{C}^{4}$, where

$$
F(x, y, z, t)=x y z+t^{2} .
$$

\footnotetext{
${ }^{2}$ This identification also explains the relation between the radial coordinate $r$ appearing in the singular KT solution, and the coordinate $\tau$ appearing in the regular KS solution (see for example Eq. (100) in Ref. [1]).
} 
The invariant variables in this function are related to the complex coordinates (2.1) by

$$
x=z_{1}^{2}, \quad y=z_{2}^{2}, \quad z=z_{3}^{2}, \quad t=\mathrm{i} z_{1} z_{2} z_{3},
$$

and thus their engineering dimensions are $[x]=[y]=[z]=L^{2}$, and $[t]=L^{3}$. The simplest deformation of the complex structure, which also resolves completely the singularity, is a constant deformation with parameter $\xi$, namely

$$
F(x, y, z, t) \rightarrow F_{\xi}(x, y, z, t)=x y z+t^{2}-\xi^{2}
$$

(notice that $[\xi]=L^{3}$ ). In Ref. [21], Berenstein has shown, via holomorphy considerations strengthened by a matrix model computation, that (5.4) is indeed the correct deformation to consider. He also showed that the deformation parameter $\xi$ is related to the gaugino condensate $S$ of the dual gauge theory, as we too will argue below. Given (5.4), we can write the holomorphic $(3,0)$-form $\Omega$ in the usual way, according to

$$
\Omega=\frac{1}{2 \pi \mathrm{i}} \oint_{F_{\xi}=0} \frac{d x \wedge d y \wedge d z \wedge d t}{F_{\xi}}=\frac{d x \wedge d y \wedge d z}{2 \sqrt{\xi^{2}-x y z}} .
$$

In order to compute the periods of $\Omega$ along a specific $A_{i}$ (or $B_{i}$ ) cycle, we define (with a little abuse of notation) $x=z_{i}^{2}, y=u+\mathrm{i} v, z=u-\mathrm{i} v$ and $\varepsilon^{2}=\xi^{2} / x$, so that from (5.5) we get

$$
\int \Omega=-\mathrm{i} \int \frac{d x \wedge d u \wedge d v}{\sqrt{\xi^{2}-x\left(u^{2}+v^{2}\right)}}=-\mathrm{i} \int \frac{d x}{\sqrt{x}} \int_{\mathcal{C}_{i}} \frac{d u \wedge d v}{\sqrt{\varepsilon^{2}-u^{2}-v^{2}}} .
$$

The last integral can be easily evaluated, and is in fact a well-known result in the context of the geometry of the K3 manifold

$$
\int_{\mathcal{C}_{i}} \frac{d u \wedge d v}{\sqrt{\varepsilon^{2}-u^{2}-v^{2}}}=\int_{-\varepsilon}^{\varepsilon} d u \int_{\gamma_{u}} \frac{d v}{\sqrt{\varepsilon^{2}-u^{2}-v^{2}}}=\int_{-\varepsilon}^{\varepsilon} d u \int_{\gamma_{\infty}} \frac{d w}{\mathrm{i} w}=4 \pi \varepsilon .
$$

Using this inside (5.6), we then have

$$
\int \Omega=-\mathrm{i} \int \frac{d x}{\sqrt{x}} 4 \pi \frac{\xi}{\sqrt{x}}=-4 \pi \mathrm{i} \xi \int \frac{d x}{x}=-8 \pi \mathrm{i} \xi \int \frac{d z_{i}}{z_{i}} .
$$

Thus, the periods of $\Omega$ along the cycles $A_{i}$ and $B_{i}$ are finally given by

$$
\int_{A_{i}} \Omega=-8 \pi \mathrm{i} \xi \oint \frac{d z_{i}}{z_{i}}=16 \pi^{2} \xi
$$

and

$$
\int_{B_{i}} \Omega=-8 \pi \mathrm{i} \xi \int_{\xi^{1 / 3}}^{\rho_{c}} \frac{d \rho_{i}}{\rho_{i}}=\frac{8 \pi \mathrm{i}}{3} \xi \ln \frac{\xi}{\rho_{c}^{3}},
$$

where in the latter we have used the same upper cutoff $\rho_{c}$ already used in the computation of the fluxes of $G_{3}$, while the lower limit of integration must now be given by a suitable 
power of the deformation parameter $\xi$ (notice that since $\xi$ has dimension $L^{3}$, this power is $1 / 3$ in order to match with the length dimension of $\left.\rho_{i}=\left|z_{i}\right|\right)$.

We have now all the necessary ingredients to compute the effective superpotential of the gauge theory by means of formula (5.1). Inserting the appropriate dimensionful prefactors, we find that the gauge effective superpotential is given by

$$
\begin{aligned}
W_{\text {eff }} & =\frac{1}{16 \pi^{2} \mathrm{i} K} \frac{1}{\left(2 \pi l_{s}^{2}\right)^{3}} \sum_{i=1}^{3}\left[\int_{A_{i}} G_{3} \int_{B_{i}} \Omega-\int_{A_{i}} \Omega \int_{B_{i}} G_{3}\right] \\
& =-\frac{1}{\left(2 \pi l_{s}^{2}\right)^{3}}\left[3\left(N-N_{f}\right) \frac{\xi}{3} \ln \frac{\xi}{\rho_{c}^{3}}+3\left(N-N_{f}\right) \xi \ln \frac{\rho_{c}}{\rho_{0}}+2 N_{f} \xi \ln \frac{\Delta}{\rho_{0}}\right] .
\end{aligned}
$$

We now re-express the geometrical quantities in terms of gauge theory quantities, by using again the "stretched string" energy/radius relation. Notice that the deformation parameter $\xi$, due to its engineering dimensions, is identified by the relation with a mass dimension 3 operator in the gauge theory, which is then natural to identify with the gaugino condensate $S$ (see also Ref. [21]). In summary the gauge/gravity relations are

$$
\rho_{c}=2 \pi l_{s}^{2} \mu, \quad \rho_{0}=2 \pi l_{s}^{2} \Lambda, \quad \Delta=2 \pi l_{s}^{2} m, \quad \xi=\left(2 \pi l_{s}^{2}\right)^{3} S,
$$

so that (5.10) becomes

$$
W_{\text {eff }}=-\left(N-N_{f}\right) S \ln \frac{S}{\Lambda^{3}}-2 N_{f} S \ln \frac{m}{\Lambda} .
$$

Though this result is correct, let us redefine the scales in order to write it in a more conventional way. The appropriate redefinition is $\Lambda \rightarrow e^{1 / 3} \Lambda, m \rightarrow e^{1 / 3} m$, and we get

$$
W_{\text {eff }}=\left(N-N_{f}\right)\left[S-S \ln \frac{S}{\Lambda^{3}}\right]-2 N_{f} S \ln \frac{m}{\Lambda},
$$

which is precisely the Taylor-Veneziano-Yankielowicz superpotential for SQCD with $N_{f}$ flavors [27. At the minimum the gaugino condensate is

$$
S=\left(\frac{\Lambda^{3 N-N_{f}}}{m^{2 N_{f}}}\right)^{\frac{1}{N-N_{f}}}
$$

so that from (5.13) we get the ADS superpotential [18]:

$$
W_{\text {eff }}=\left(N-N_{f}\right)\left[\frac{\Lambda^{3 N-N_{f}}}{m^{2 N_{f}}}\right]^{\frac{1}{N-N_{f}}}=\left(N-N_{f}\right)\left[\frac{\Lambda^{3 N-N_{f}}}{\operatorname{det} M}\right]^{\frac{1}{N-N_{f}}} .
$$

As an aside, we note that if $N_{f}=0$ the above results reproduce the Veneziano-Yankielowicz superpotential for pure $\mathcal{N}=1$ Super Yang-Mills theory [25]

$$
W_{\mathrm{VY}}=N\left[S-S \ln \frac{S}{\Lambda^{3}}\right] \text {. }
$$


Its value at the minimum (where $S=\Lambda^{3}$ ) is $W_{\mathrm{VY}}=N \Lambda^{3}$. We stress that this is not just a formal limit of the result obtained for $N_{f}>0$; indeed, one could have started from the beginning by considering only $N$ fractional branes of type A, and apply formula (5.1) to obtain the Veneziano-Yankielowicz superpotential.

Another observation concerns the case in which $N_{f}=N$. As we have mentioned before, our brane construction can still be used in this case where all branes form $\mathrm{A}+\mathrm{B}$ superpositions and no fractional branes (and thus no effective gauge theory) are left. Therefore, the result for the moduli space can be read from the superpotential (5.13) for $N_{f}=N$. In

this case, the minimization procedure implies $\operatorname{det} M=\Lambda^{2 N}$, which is indeed the correct result expected for a $\mathcal{N}=1$ gauge theory with gauge group $U(N)$ and $N$ flavors.

Therefore we can conclude that our classical supergravity solution, together with some geometrical considerations, has been able to provide relevant information on the $\mathcal{N}=1$ SQCD with $N_{f}$ flavors, both at at the classical and perturbative level, and also at a nonperturbative level. It would be very interesting to use this system of fractional branes to analyze SQCD also in the phase where $N_{f}>N$, where Seiberg duality is supposed to take place [28]. Indeed a construction of Seiberg duality for generic quiver theories was presented in Refs. [29, 21] with the implementation of quite formal methods involving also anti-branes. A more explicit analysis of these methods in the specific model we have studied here is under current investigation [30].

\section{Acknowledgments}

We would like to thank Matteo Bertolini, Marco Billò, Paolo Di Vecchia, Paolo Merlatti, Christian Römelsberger and Giuseppe Vallone for insightful discussions. E.I. also acknowledges feedback received at the Simons Workshop in Mathematics and Physics held at Stony Brook in August-September 2003 and at the RTN Workshop held in Copenhagen in September 2003. This work is partially supported by the European Commission RTN programme HPRN-CT-2000-00131, and by MIUR under contract 2001-1025492.

\section{References}

[1] I. R. Klebanov and N. A. Nekrasov, "Gravity duals of fractional branes and logarithmic RG flow," Nucl. Phys. B574 (2000) 263-274, hep-th/9911096.

[2] I. R. Klebanov and A. A. Tseytlin, "Gravity duals of supersymmetric $S U(N) \times S U(N+$ M) gauge theories," Nucl. Phys. B578 (2000) 123-138, hep-th/0002159. 
[3] I. R. Klebanov and M. J. Strassler, "Supergravity and a confining gauge theory: Duality cascades and $\chi$ SB-resolution of naked singularities," JHEP 08 (2000) 052, hep-th/0007191.

[4] A. Loewy and J. Sonnenschein, "On the holographic duals of $\mathcal{N}=1$ gauge dynamics," JHEP 08 (2001) 007, hep-th/0103163.

[5] E. Imeroni, "On the $\mathcal{N}=1 \beta$-function from the conifold," Phys. Lett. B541 (2002) 189-193, hep-th/0205216

[6] M. Bertolini, P. Di Vecchia, M. Frau, A. Lerda, R. Marotta, and I. Pesando, "Fractional D-branes and their gauge duals," JHEP 02 (2001) 014, hep-th/0011077; J. Polchinski, "N $\mathcal{N}=2$ gauge-gravity duals," Int. J. Mod. Phys. A16 (2001) 707-718, hep-th/0011193; M. Bertolini, P. Di Vecchia, M. Frau, A. Lerda, and R. Marotta, "N $=2$ gauge theories on systems of fractional D3/D7 branes," Nucl. Phys. B621 (2002) 157-178, hep-th/0107057; M. Billò, L. Gallot, and A. Liccardo, "Classical geometry and gauge duals for fractional branes on ALE orbifolds," Nucl. Phys. B614 (2001) 254-278, hep-th/0105258 M. Bertolini, P. Di Vecchia, M. Frau, A. Lerda, and R. Marotta, "More anomalies from fractional branes," Phys. Lett. B540 (2002) 104-110, hep-th/0202195 P. Di Vecchia, A. Liccardo, R. Marotta, and F. Pezzella, "Gauge/gravity correspondence from open/closed string duality," JHEP 06 (2003) 007, hep-th/0305061.

[7] M. Bertolini, P. Di Vecchia, G. Ferretti, and R. Marotta, "Fractional branes and $\mathcal{N}=1$ gauge theories," Nucl. Phys. B630 (2002) 222-240, hep-th/0112187

[8] R. Marotta, F. Nicodemi, R. Pettorino, F. Pezzella, and F. Sannino, "N $\mathcal{N}=1$ matter from fractional branes," JHEP 09 (2002) 010, hep-th/0208153.

[9] J. M. Maldacena and C. Núñez, "Supergravity description of field theories on curved manifolds and a no go theorem," Int. J. Mod. Phys. A16 (2001) 822-855, hep-th/0007018; J. M. Maldacena and C. Núñez, "Towards the large $N$ limit of pure $\mathcal{N}=1$ super Yang Mills," Phys. Rev. Lett. 86 (2001) 588-591, hep-th/0008001.

[10] J. P. Gauntlett, N. Kim, D. Martelli, and D. Waldram, "Wrapped fivebranes and $\mathcal{N}=2$ super Yang-Mills theory," Phys. Rev. D64 (2001) 106008, hep-th/0106117; F. Bigazzi, A. L. Cotrone, and A. Zaffaroni, " $\mathcal{N}=2$ gauge theories from wrapped fivebranes," Phys. Lett. B519 (2001) 269-276, hep-th/0106160 R. Apreda, F. Bigazzi, A. L. Cotrone, M. Petrini, and A. Zaffaroni, "Some comments on $\mathcal{N}=1$ gauge theories from wrapped branes," Phys. Lett. B536 (2002) 161-168, hep-th/0112236; P. Di Vecchia, A. Lerda, and P. Merlatti, " $\mathcal{N}=1$ and $\mathcal{N}=2$ super Yang-Mills theories from wrapped branes," Nucl. Phys. B646 (2002) 43-68, hep-th/0205204; 
M. Bertolini and P. Merlatti, "A note on the dual of $\mathcal{N}=1$ super Yang-Mills theory," Phys. Lett. B556 (2003) 80-86, hep-th/0211142 W. Mück, "Perturbative and nonperturbative aspects of pure $\mathcal{N}=1$ super Yang-Mills theory from wrapped branes," JHEP 0302, 013 (2003) hep-th/0301171.

[11] C. P. Herzog, I. R. Klebanov, and P. Ouyang, "D-branes on the conifold and $\mathcal{N}=1$ gauge/gravity dualities," hep-th/0205100.

[12] M. Bertolini, "Four lectures on the gauge-gravity correspondence," hep-th/0303160; F. Bigazzi, A. L. Cotrone, M. Petrini, and A. Zaffaroni, "Supergravity duals of supersymmetric four dimensional gauge theories," Riv. Nuovo Cim. 25N12 (2002) 1-70, hep-th/0303191; P. Di Vecchia and A. Liccardo, "Gauge theories from D-branes," hep-th/0307104.

[13] R. Gopakumar and C. Vafa, "On the gauge theory/geometry correspondence," $A d v$. Theor. Math. Phys. 3 (1999) 1415-1443, hep-th/9811131.

[14] C. Vafa, "Superstrings and topological strings at large N," J. Math. Phys. 42 (2001) 2798-2817, hep-th/0008142.

[15] F. Cachazo, K. A. Intriligator, and C. Vafa, "A large $N$ duality via a geometric transition," Nucl. Phys. B603 (2001) 3-41, hep-th/0103067.

[16] F. Cachazo, S. Katz, and C. Vafa, "Geometric transitions and $\mathcal{N}=1$ quiver theories," hep-th/0108120; F. Cachazo, B. Fiol, K. A. Intriligator, S. Katz, and C. Vafa, "A geometric unification of dualities," Nucl. Phys. B628 (2002) 3-78, hep-th/0110028.

[17] T. R. Taylor and C. Vafa, "RR flux on Calabi-Yau and partial supersymmetry breaking," Phys. Lett. B474 (2000) 130-137, hep-th/9912152.

[18] I. Affleck, M. Dine, and N. Seiberg, "Dynamical Supersymmetry Breaking In Supersymmetric QCD," Nucl. Phys. B241 (1984) 493-534.

[19] M. R. Douglas, B. R. Greene, and D. R. Morrison, "Orbifold resolution by D-branes," Nucl. Phys. B506 (1997) 84-106, hep-th/9704151.

[20] M. R. Douglas and G. W. Moore, "D-branes, Quivers, and ALE Instantons," hep-th/9603167.

[21] D. Berenstein, "D-brane realizations of runaway behavior and moduli stabilization," hep-th/0303230.

[22] C. V. Johnson, A. W. Peet, and J. Polchinski, "Gauge theory and the excision of repulson singularities," Phys. Rev. D61 (2000) 086001, hep-th/9911161. 
[23] A. W. Peet and J. Polchinski, "UV/IR relations in AdS dynamics," Phys. Rev. D59 (1999) 065011, hep-th/9809022.

[24] K. A. Intriligator and N. Seiberg, "Lectures on supersymmetric gauge theories and electric-magnetic duality," Nucl. Phys. Proc. Suppl. 45BC (1996) 1-28, hep-th/9509066.

[25] G. Veneziano and S. Yankielowicz, "An effective Lagrangian for the pure $\mathcal{N}=1$ supersymmetric Yang-Mills theory," Phys. Lett. B113 (1982) 231.

[26] S. B. Giddings, S. Kachru, and J. Polchinski, "Hierarchies from fluxes in string compactifications," Phys. Rev. D66 (2002) 106006, hep-th/0105097.

[27] T. R. Taylor, G. Veneziano, and S. Yankielowicz, "Supersymmetric QCD and its massless limit: An effective Lagrangian analysis," Nucl. Phys. B218 (1983) 493.

[28] N. Seiberg, "Electric-magnetic duality in supersymmetric non-Abelian gauge theories," Nucl. Phys. B435 (1995) 129-146, hep-th/9411149.

[29] D. Berenstein and M. R. Douglas, "Seiberg duality for quiver gauge theories," hep-th/0207027.

[30] Work in progress. 